CERN-EP-2000-039

14 February 2000

\title{
Dependence of Drift Tube Performance on the Anode Wire Diameter
}

\author{
M. Deile ${ }^{1)}$, M. Aleksa ${ }^{2)}$, J. Dubbert ${ }^{1)}$, C.W. Fabjan ${ }^{2)}$, C. Gruhn ${ }^{3)}$, N.P. Hessey ${ }^{1, a)}$, \\ W. Riegler ${ }^{2, b)}$, T. Sammer ${ }^{1, c)}$ and A. Staude ${ }^{1)}$
}

\begin{abstract}
Cylindrical pressurized drift tubes with different anode wire diameters were operated in a $170 \mathrm{GeV}$ muon test beam. The dependences of spatial resolution, efficiency and streamer probability on the anode wire diameter were measured. The resolution measurements are compared with a simulation.
\end{abstract}

(Submitted to Nuclear Instruments and Methods)

\footnotetext{
1) Ludwig-Maximilians-Universität München, Sektion Physik, Lehrstuhl Schaile, Am Coulombwall 1, 85748 Garching, Germany

2) EP Division, CERN, CH-1211 Genève 23, Switzerland

3) Max-Planck-Institut für Physik, Föhringer Ring 6, 80805 München, Germany

a) now at NIKHEF, Kruislaan 409, 1098 SJ Amsterdam, Netherlands

b) now at Harvard University, 42 Oxford Street, Cambridge MA 02138, USA

c) now at Max-Planck-Institut für Physik, Föhringer Ring 6, 80805 München, Germany
} 
The precision chambers of the ATLAS muon spectrometer [1, 2] will consist of 1200 chambers with multilayers of pressurized drift tubes. In order to achieve the goals defined by the physics programme, a relative momentum resolution of $10 \%$ is required for $1 \mathrm{TeV}$ muons. Given the toroidal magnetic field of about $0.5 \mathrm{~T}$ over a typical track length of $5 \mathrm{~m}$ in the spectrometer, this requires a spatial resolution of about $80 \mu \mathrm{m}$ for a single drift tube. To meet this ambitious specification, detailed studies of the operating parameters had to be performed (see e.g. [3]). We found as optimum, considering mainly resolution and total accumulated charge during the life time of the experiment, to operate the ATLAS drift tubes in proportional mode with a low gas amplification of $2 \cdot 10^{4}$ and 3 bar drift-gas pressure. In order to avoid noise hits, the discriminator threshold will be set to 5 times the rms noise level.

The present article focuses on the impact of the anode wire diameter on the drift tube performance. The main subjects of the measurements were resolution and efficiency. In addition the fraction of limited streamer signals was investigated. Since in limited streamer mode the charge deposit is up to 100 times higher than in proportional mode, the streamer fraction has to be kept low to avoid ageing problems.

The choice of the anode wire diameter is not only driven by performance considerations, but also by the need for good mechanical strength. Therefore the decision was in favour of the thickest wire diameter which still complies with the physics requirements.

\section{$2 \quad$ Experimental Setup}

The measurement setup is shown in Figure 1.

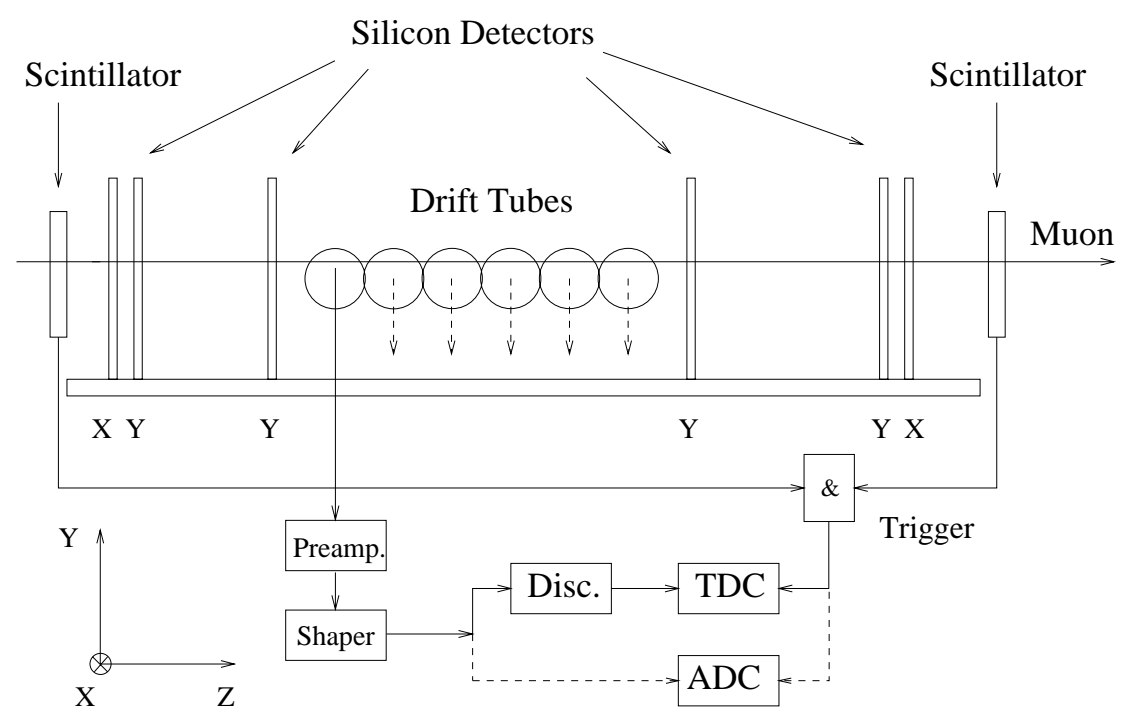

Figure 1: Experimental setup

Five drift tubes with different wire diameters were operated in the halo of the SPS beamline M2 at CERN. The muon energy was about $170 \mathrm{GeV}$. Table 1 shows a summary of the fundamental properties and operating conditions of these drift tubes. Parameters specific to the wire diameter are given in Table 2.

Our measurements were made before the final decision on the baseline gas for ATLAS (Ar/ $\mathrm{CO}_{2}$ 93/7 [4]). Also the anode wire material was different from the one now chosen (gold-plated W/Re 97/3). We assume however that our conclusions about the 
dependence of drift-tube performance on the wire diameter will not be affected by these changes.

\begin{tabular}{|l|l|}
\hline inner tube radius & $14.6 \mathrm{~mm}$ \\
outer tube radius & $15.0 \mathrm{~mm}$ \\
tube length & $1 \mathrm{~m}$ \\
anode wire material & $\mathrm{Cu} / \mathrm{Be}(50,70,100,150 \mu \mathrm{m})$ \\
& $\mathrm{INOX}(30 \mu \mathrm{m})$ \\
gas & $\mathrm{Ar} / \mathrm{N}_{2} / \mathrm{CH}_{4} 91 / 4 / 5[5]$ \\
pressure & $3 \mathrm{bar}$ absolute \\
gas gain & $(1,2,6) \cdot 10^{4}$ \\
maximum drift time & $480 \mathrm{~ns}$ \\
peaking time of the shaper & $15 \mathrm{~ns}$ \\
discriminator threshold & $5 \times \mathrm{rms}$ noise \\
\hline
\end{tabular}

Table 1: General parameters of the drift tubes in the test setup

\begin{tabular}{|c|c|c|c|}
\hline $\begin{array}{c}\text { Wire } \\
\text { diameter }[\mu \mathrm{m}]\end{array}$ & $\begin{array}{c}\text { Tube } \\
\text { impedance }[\Omega]\end{array}$ & $\begin{array}{c}\text { Voltage }[\mathrm{V}] \\
\text { for } G=2 \cdot 10^{4}\end{array}$ \\
\hline 30 & 413 & 2750 & 10.5 \\
50 & 382 & 3280 & 16.3 \\
70 & 362 & 3760 & 20.6 \\
100 & 340 & 4335 & 27.2 \\
150 & 316 & 4960 & 42.2 \\
\hline
\end{tabular}

Table 2: Tube and operating parameters depending on the wire diameter

One end of each tube was read out with a current sensitive preamplifier, the other end was terminated with a resistor matching the tube impedance $Z_{\text {tube }}$ in order to avoid signal reflections. $Z_{\text {tube }}$ is given by

$$
Z_{\text {tube }}=\sqrt{\frac{L}{C}}=\frac{1}{2 \pi} \sqrt{\frac{\mu}{\varepsilon}} \ln \frac{b}{a},
$$

where $L$ and $C$ are the inductance and capacitance per unit tube length, $a$ and $b$ the radii of the wire and the inner tube wall, $\mu$ the magnetic permeability and $\varepsilon$ the permittivity of the gas. The values of $Z_{\text {tube }}$ for the tubes under study are given in Table 2 .

For the resolution and efficiency measurements an amplifier/shaper combination [6] with $15 \mathrm{~ns}$ peaking time was used. The output was connected to a discriminator and a TDC with a time binning of $1 \mathrm{~ns}$. The discriminator threshold was set to $5 \times$ the rms noise voltage, which is dominated by the thermal noise of the termination resistor. Table 2 gives the threshold in units of primary electrons (p.e.), i.e. as multiples of the pulse height corresponding to a single ionization electron The strong dependence on the wire diameter will be explained in Section 3 .

For the calibration of the relationship between voltage and gas gain (Figure 2 and Table 2) a preamplifier with 4 ns shaping time [7] and a charge sensitive ADC were used. The same setup served for the streamer-rate studies. The gas gain and streamer rates were measured with a set of tubes containing a $160 \mu \mathrm{m}$ wire instead of the $150 \mu \mathrm{m}$ wire; 


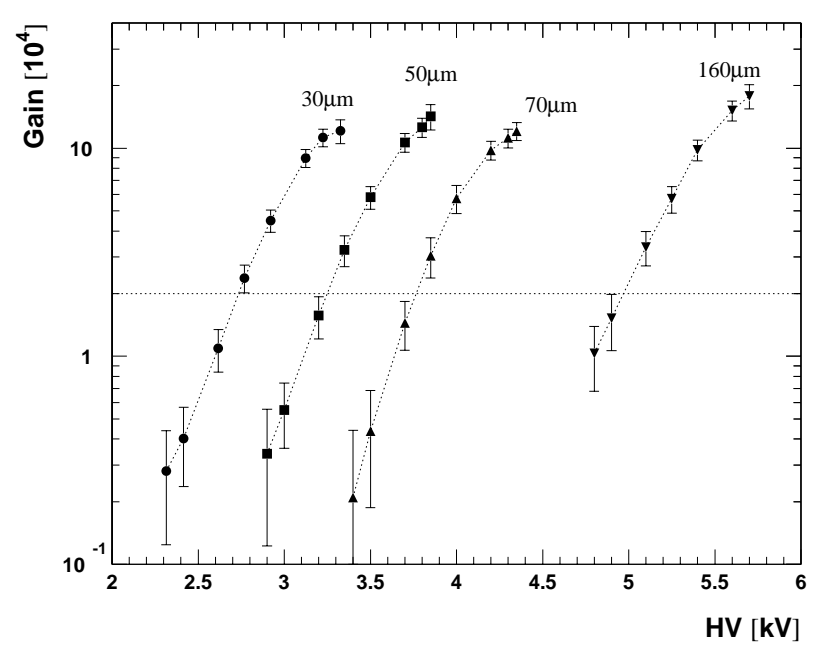

Figure 2: Gas gain of $\mathrm{Ar} / \mathrm{N}_{2} / \mathrm{CH}_{4} 91 / 4 / 5$ in proportional mode as a function of the anode voltage for different wire diameters. The connecting lines are drawn to guide the eye.

no $100 \mu \mathrm{m}$ wire was available. In Table 2 the voltages for $100 \mu \mathrm{m}$ and $150 \mu \mathrm{m}$ have been interpolated. Details about the gas gain measurement are described in [5].

As external reference system we used a tracker consisting of six Silicon microstrip detectors with $7 \mu \mathrm{m}$ resolution each. Four out of the six detectors measured the precision coordinate perpendicular to the tube wires, the other two gave the second coordinate parallel to the wires. The resolution for the track position at the chamber was $5 \mu \mathrm{m}$, somewhat greater than $\frac{7 \mu \mathrm{m}}{\sqrt{4}}$ due to multiple scattering $[8,9]$.

The experiment was triggered by two scintillators covering the sensitive area of the silicon detectors $\left(50 \times 50 \mathrm{~mm}^{2}\right)$. 
Figure 3 shows the space-time distribution for the tube with the $50 \mu \mathrm{m}$ wire. The space coordinate $r$ is the minimum distance of the muon track from the anode wire as given by the silicon tracker. The drift time $t$ is measured by the TDC. The entries below the "V"-shaped band correspond to electromagnetic secondaries (mainly delta-rays) produced by the muon: if a delta-ray is emitted towards the anode wire of the tube, its own ionization signal can hide the muon pulse, and the measured drift time is too short.

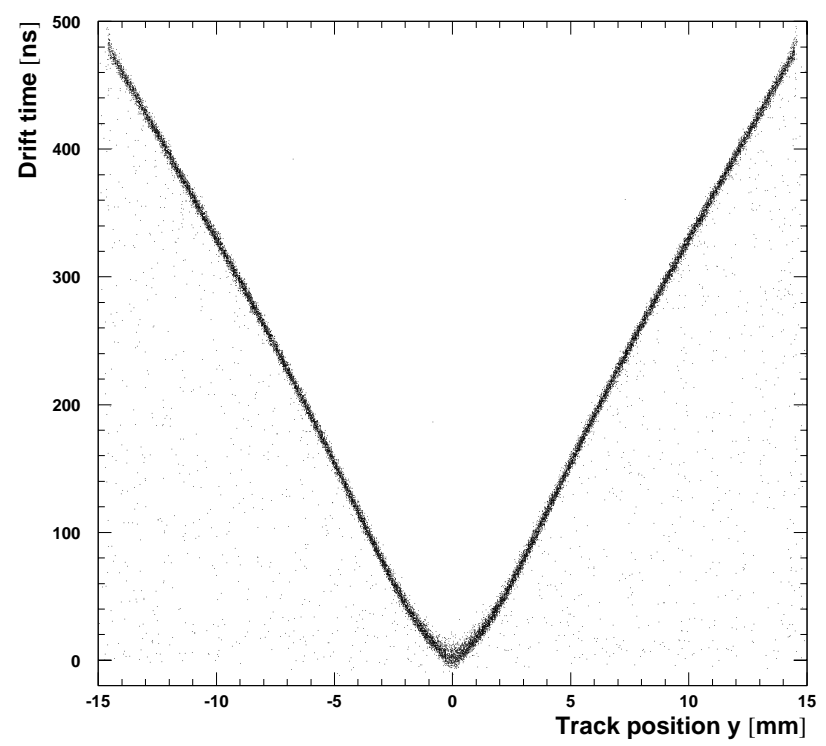

Figure 3: Drift time measured with the TDC versus track distance from the anode wire given by the silicon tracker.

The r-t relationship which is used for reconstructing track radii, is defined as the center of the band in Figure 3. The resolution corresponds to the width of this band. It is determined together with the r-t relationship in the following iterative way: We start with a first-guess r-t relationship $\rho_{0}(t)$ and plot the residuals $\Delta r=\rho_{0}\left(t_{\text {drift }}\right)-r_{\text {silicon }}$ as a function of the drift time $t_{\text {drift }}$, where $r_{\text {silicon }}$ is the track radius given by the silicon tracker. Then we project time slices of this 2-dimensional distribution onto the $\Delta r$ axis and fit them with Gaussians. The means of these Gaussian fits are used as corrections to $\rho_{0}(t)$ which yields a better estimate $\rho_{1}(t)$. After about 5 iterations the residual distribution converges at 0 (Figure 4). Its Gaussian width is defined as the resolution. Far from the wire this Gaussian approximation is quite good whereas for $r<2 \mathrm{~mm}$ the cross-sections of the r-t-distribution have considerable non-gaussian tails due to the cluster statistics of the ionization process [10]. Deviations from the Gaussian shape are the main contribution to the error of the resolution measurement because the width of the Gaussian fit depends on the range around the maximum in which the fit is done. It was empirically found that acceptable fits are obtained for a range of 2 standard deviations around the peak.

Figure 6 shows the resolution as a function of the track radius for each wire diameter studied. In addition to the measurements a simulation with the program GARFIELD [11] is displayed. At every millimeter of radial distance from the wire 2000 muon tracks were simulated. The ionization of the muons was generated according to the exponential cluster-distance distribution and the cluster-size distribution calculated with the program 


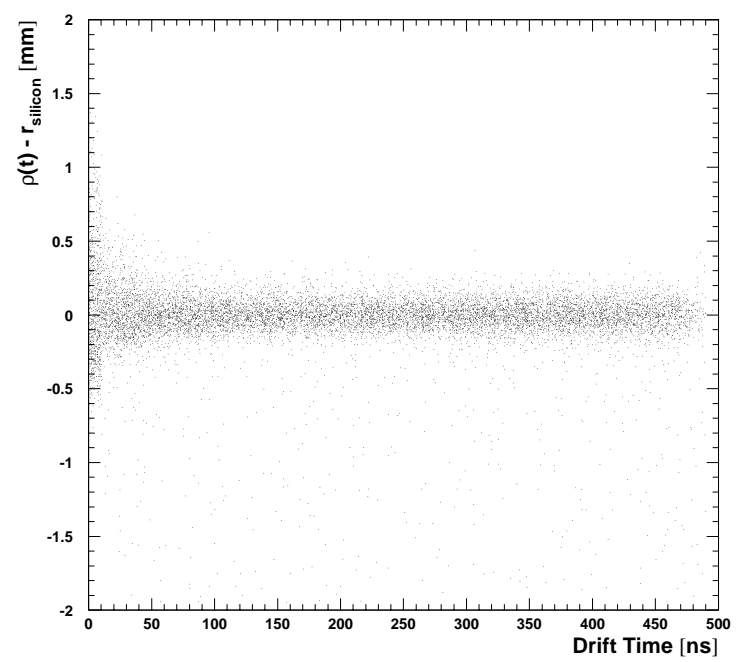

Figure 4: Residuals $\rho\left(t_{\text {drift }}\right)-r_{\text {silicon }}$ versus drift time at the end of the iterative determination of the r-t relationship $\rho(t)$.
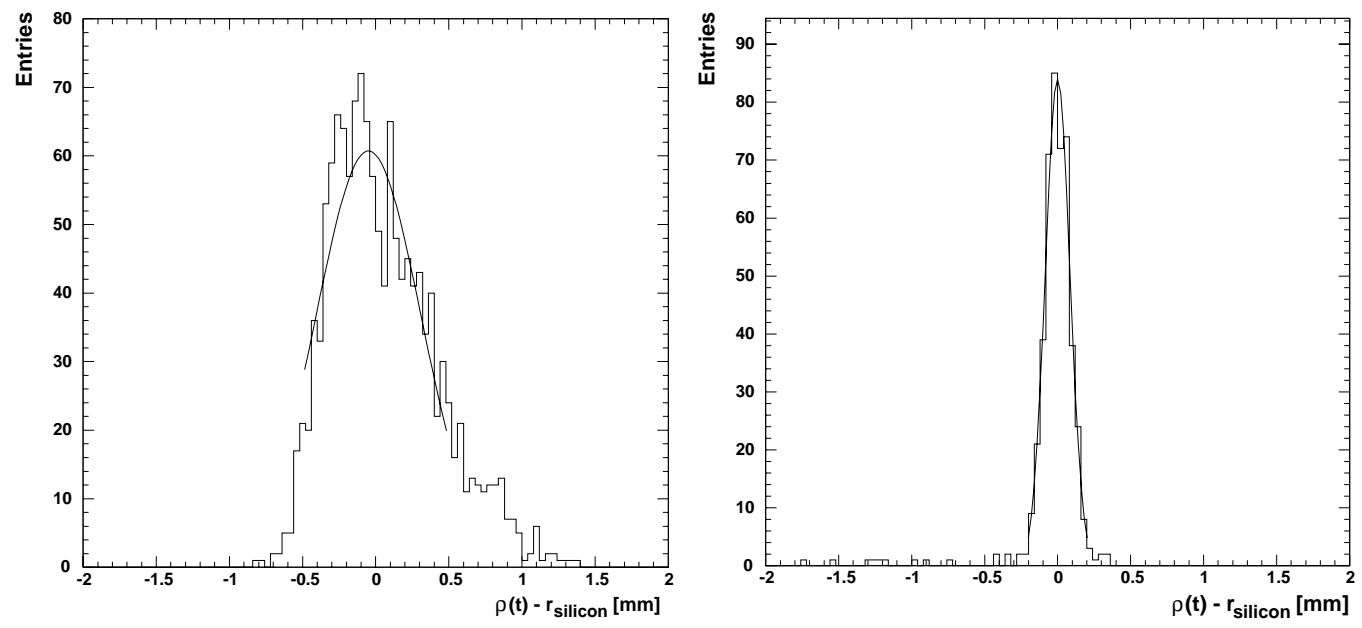

Figure 5: Projections of time slices of Figure 4. Left: $0<t<10 \mathrm{~ns}$; right: $100 \mathrm{~ns}<t<$ 110 ns. Superimposed are Gaussian fits to a range of 2 standard deviations around the peak.

HEED [12] which is interfaced to GARFIELD. Then the drift of the ionization electrons was simulated using the transport properties of the gas calculated with MAGBOLTZ [13] which is also interfaced to GARFIELD. The charge amplification process near the anode wire was modelled with a Polya distribution. Then the signal induced on the wire by the drift of the positive ions from the wire to the wall was calculated. Finally the signal was convoluted with the delta-response of the read-out electronics, and the threshold-crossing time was determined.

For the ion drift the field-dependent mobility of $\mathrm{Ar}^{+}$in $\mathrm{Ar}$ was used. This is a simplification because in reality charge transfer processes take place, and not only $\mathrm{Ar}^{+}$ ions but also other ions like $\left[\mathrm{CH}_{4}\right]^{+}$are drifting. Their mobility might be different. Also not taken into account are the sharp electron pulse before the ion pulse and the fact that 

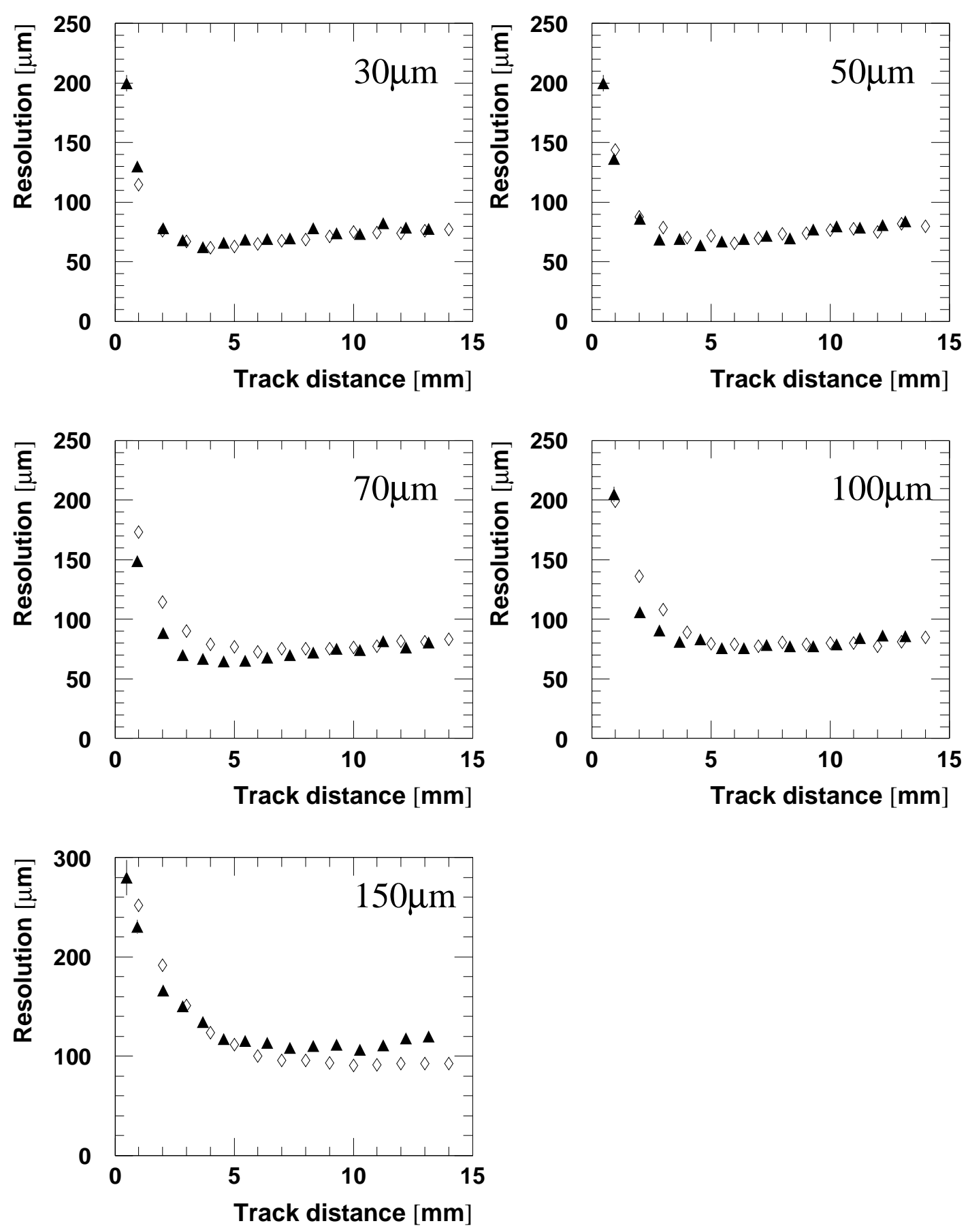

Figure 6: Spatial resolution as a function of the distance of the muon track from the wire for different wire diameters. The gas gain was $2 \cdot 10^{4}$. The triangles represent the measurements, the diamonds show a simulation using GARFIELD [11]. The errors are typically between $5 \mu \mathrm{m}$ (far from the wire) and $10 \mu \mathrm{m}$ (near the wire, where the resolution is non-gaussian). 
the ions are not produced exactly on the wire surface but in the whole avalanche region. The details of the amplification process are very difficult to simulate. Nevertheless the simulation is in fairly good agreement with the measurements.

To facilitate the resolution comparison of the wires with different diameters, we calculate the quadratic mean resolution

$$
<\sigma>\equiv \sqrt{\frac{1}{R} \int_{1 \mathrm{~mm}}^{R}[\sigma(r)]^{2} d r} .
$$

Following the convention in [1], the first millimeter is excluded from the average because near the wire the resolution is so bad that hits in this range will often not be taken into account by track fits through an MDT chamber. Furthermore, the quadratic average would be dominated by the large $\sigma(r)$ in this small interval and would not be a representative number. The results are presented in Figure 7. In addition to our measurements (solid markers) a Garfield simulation is shown.

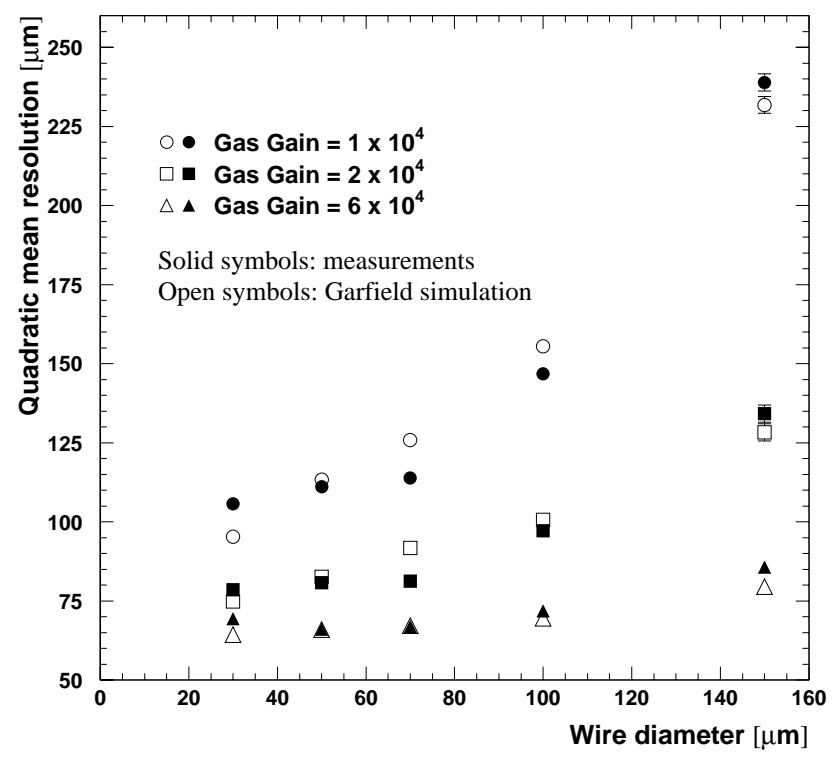

Figure 7: Dependence of the quadratic mean resolution as defined in (2) on the wire diameter for different gas gains.

The resolution deterioration with increasing wire diameter is a consequence of the higher threshold in terms of primary electrons (see Table 2): In [3] we have shown that the resolution is approximately proportional to the trigger electron $n_{t h r}$ which is given by

$$
n_{t h r}=\frac{V_{t h r}}{V_{1 e}}
$$

where $V_{t h r}$ is the threshold voltage and $V_{1 e}$ the height of a single primary electron pulse at the output of the shaper. To avoid noise hits, $V_{t h r}$ is required to be 5 times the rms noise voltage, which is dominated by the thermal Equivalent Noise Charge (ENC) of the termination resistor $R_{t}=Z_{\text {tube }}$ at the high-voltage end of the tube:

$$
E N C=\sqrt{\frac{k T}{R_{t}} \tau},
$$


where $k$ is the Boltzmann constant, $T$ the temperature and $\tau=15$ ns the preamplifier peaking time [3]. Together with equation (1) follows

$$
E N C \sim \frac{1}{\sqrt{\ln \frac{b}{a}}},
$$

i.e. the noise decreases slowly with decreasing wire diameter $a$.

The height $V_{1 e}$ of the pulse created by a single ionization electron is proportional to

$$
I_{1 e}=\frac{G e \mu V}{a^{2}\left(\ln \frac{b}{a}\right)^{2}}
$$

with the gas gain $G$, the anode voltage $V$ and the ion mobility $\mu$ which is approximated to be independent of the electric field [14]. Thus the signal height increases with decreasing wire diameter $a$.

From (3), (5) and (6) follows

$$
n_{t h r} \sim \frac{a^{2}}{G} \cdot \frac{\left(\ln \frac{b}{a}\right)^{\frac{3}{2}}}{V(G, a)}
$$

The dependence of the operation voltage $V(G, a)$ on both the gain and the wire radius is less than linear (visible in Figure 2). Therefore one expects the trigger electron and thus the resolution to have approximately a quadratic dependence on the wire radius $a$ and an inverse proportionality to the gain $G$, which is in qualitative agreement with the measurements and the simulation. However, the saturation of the resolution for decreasing wire diameter is more accentuated in the measurements than in the simulation.

The important conclusion for the development of ATLAS drift tubes is that from the point of view of resolution optimization there is no advantage in choosing a wire diameter thinner than $50 \mu \mathrm{m}$. 
The efficiency of drift tubes is not expected to have a significant dependence on the wire diameter, and indeed the measurement didn't show any dependence. However for a more complete description of drift tube performance we present our efficiency measurements in this section.

Drift tube efficiency has two aspects: The first one is the simple probability for detecting a crossing particle at all; we call it the hit efficiency which is described in Section 4.1. For pattern recognition in a high-rate background it is important to have a very high hit efficiency.

To get a relevant efficiency for track reconstruction it is appropriate to apply a quality cut on the detected hits, because a reconstructed hit radius which is far from the true impact radius doesn't provide any information for the track fit. Therefore we introduce the so-called $3 \sigma$-efficiency which is discussed in Section 4.2.

\subsection{Hit Efficiency}

The hit efficiency $\eta_{h i t}$ is defined as the fraction of events with a hit registered in the total drift time window, when the silicon tracker predicts that a track passed through the tube.
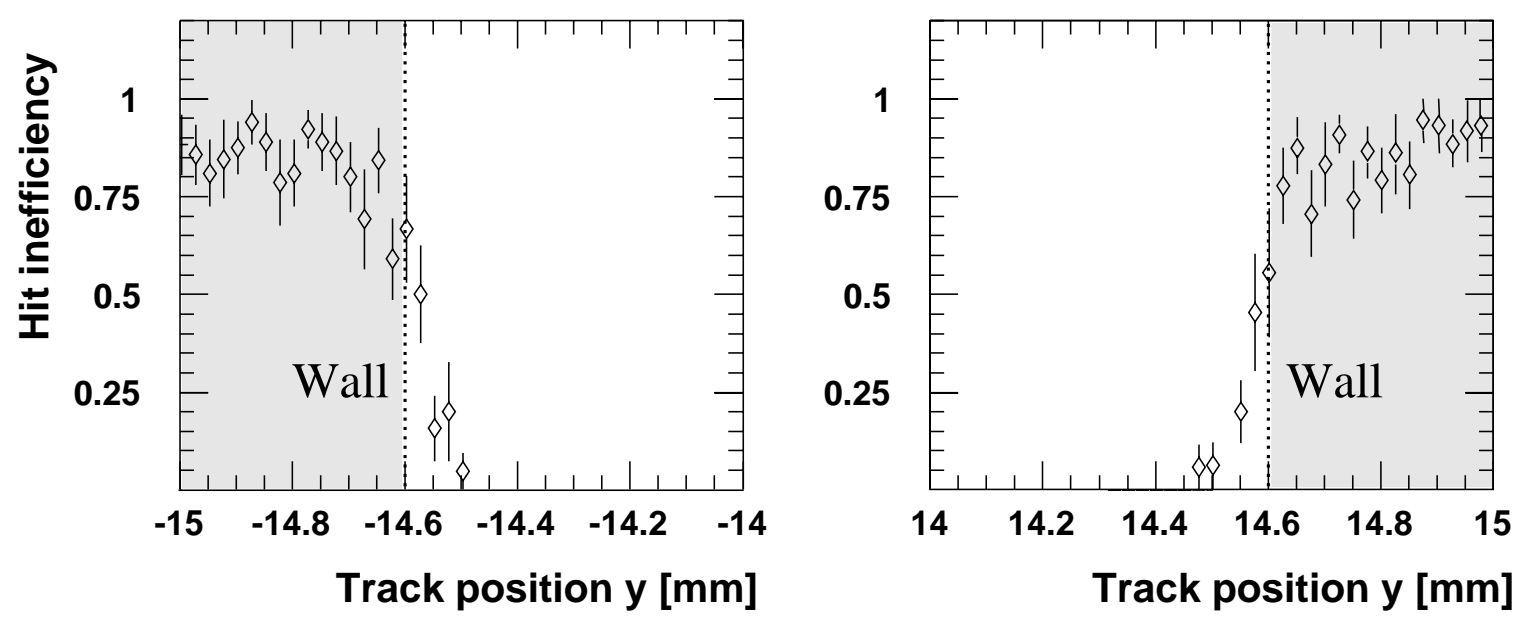

Figure 8: Hit inefficiency at the tube walls for a gas gain of $2 \times 10^{4}$ and a wire diameter of $50 \mu \mathrm{m}$. The other tubes with different wire diameters show the same behaviour.

The measurement showed for all tubes that the inefficiency is only significant for tracks within $200 \mu \mathrm{m}$ of the wall (Figure 8). This is due to the fact that near the wall the track length within the gas volume becomes short and in some cases the primary ionization is too small for obtaining a pulse greater than the discriminator threshold. The figure also demonstrates that even some tracks passing only through the aluminium wall without entering the gas volume are detected, leading to an inefficiency $<1$ for $r>14.6 \mathrm{~mm}$. This effect is caused by delta-rays knocked out of the aluminium and flying into the gas volume where they create an ionization signal.

In order to separate the geometry-related inefficiency at the tube walls from the inefficiency of the gas volume, we apply a cut at $r=14.4 \mathrm{~mm}$ and consider only the efficiency inside this radius. The resulting hit inefficiency is only

$$
1-\eta_{h i t}=(4.30 \pm 4.28) \times 10^{-5}
$$


i.e. 1 missed hit in 23000 events. The almost perfect efficiency can be understood taking into consideration the high primary ionization of about 700 electrons in our argon-based gas mixture at a pressure of 3 bar absolute and the low discriminator threshold of less than 50 electrons for all wires.

As expected, we did not see any dependence of the hit efficiency on the wire diameter.

\section{$4.23 \sigma$-Efficiency}

As a quality criterion for muon hit reconstruction we introduce a $3 \sigma$-cut and define the $3 \sigma$-efficiency by

$$
\eta_{3 \sigma}=\frac{\text { number of hits reconstructed with } \rho(t)-r_{\text {silicon }}<3 \sigma\left(r_{\text {silicon }}\right)}{\text { number of tracks through the tube }} .
$$

Again the silicon tracker is used as external reference. Since $\sigma\left(r_{\text {silicon }}\right)$ is the Gaussian approximation to the spatial resolution of the tube at the distance $r_{\text {silicon }}$ from the wire, $\eta_{3 \sigma}$ quantifies the importance of non-gaussian tails in the drift-time distribution for a given radius. Figure 9 shows $\eta_{3 \sigma}$ as a function of $r$.

The decrease of $\eta_{3 \sigma}$ from the tube center towards the wall can be explained by the increasing probability for the muon being hidden by a delta-ray (see Figure 3) creating a hit outside $3 \sigma$.

The little hollow near the wire is a consequence of the non-gaussian errors due to clustering effects which are important in that domain (see Figure 5b).

As with the hit efficiency, there is no visible dependence of $\eta_{3 \sigma}$ on the wire diameter.

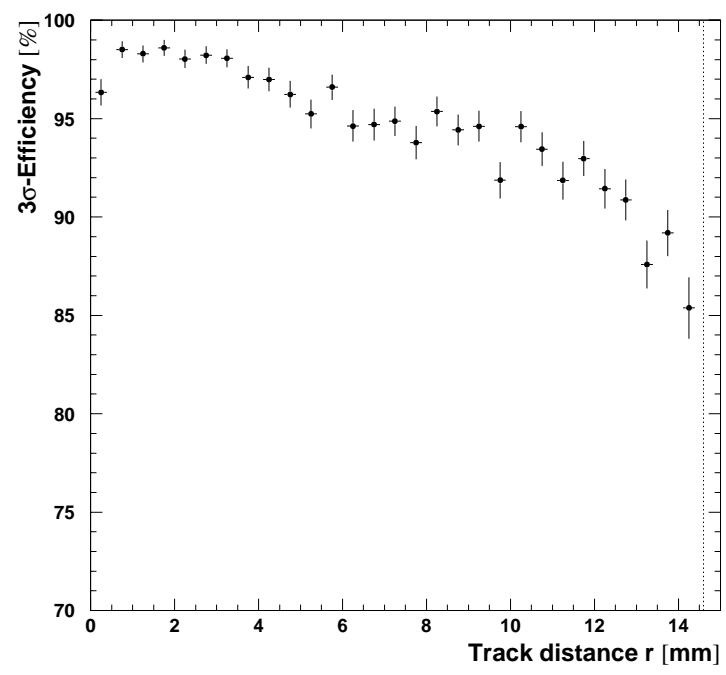

Figure 9: $3 \sigma$-Efficiency for a gas gain of $2 \times 10^{4}$ and a wire diameter of $50 \mu \mathrm{m}$. The results for the other wires are identical within the error bars. 
To minimize ageing, ATLAS drift tubes have to be operated in proportional mode. Since streamer pulses deposit up to 100 times the charge of a proportional pulse, a streamer fraction of $1 \%$ could double the total deposited charge. The ATLAS collaboration defined $1 \%$ as the upper limit [15].

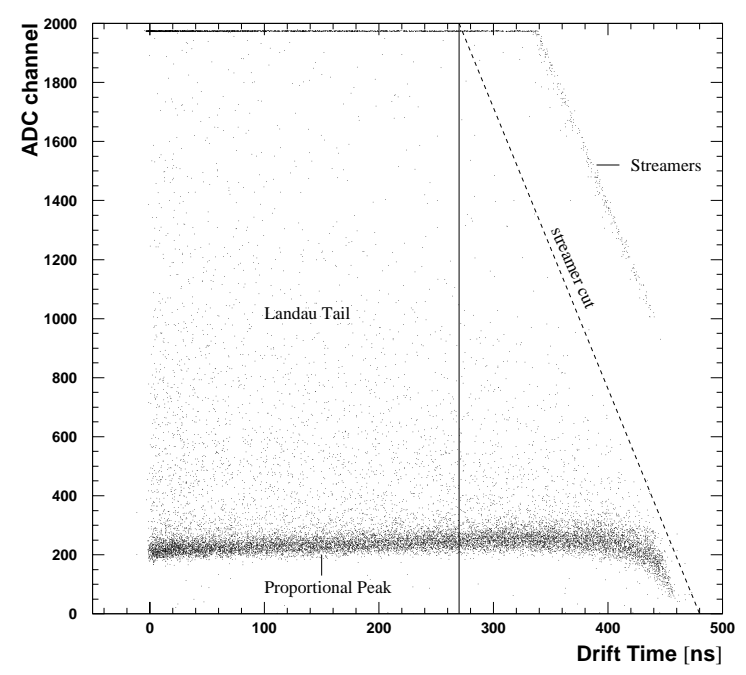

Figure 10: ADC channel (proportional to the charge) versus drift time for the $70 \mu \mathrm{m}$ wire at $4.2 \mathrm{kV}$.

To distinguish streamer pulses from events in the Landau tail of signals in proportional mode, a feature of our read-out electronics was used (Figure 10): Generally the charge of a streamer pulse is far beyond the range of our ADC, and their entries in the ADC spectrum lie in the overflow together with events belonging to the Landau tail. However, the gate of the ADC was only $600 \mathrm{~ns}$ long. Thus signals with long drift times are not integrated over the whole gate length, and even streamers remain in the range of the ADC. In Figure 10 this mechanism produces, for drift times greater than about $270 \mathrm{~ns}$, a clear gap between proportional and streamer signals, the latter visible as a narrow band due to amplifier saturation. Therefore the streamer-pulse probability was determined by counting the entries above and below the cut line for drift times $>270 \mathrm{~ns}$. This limit biases us to tracks far from the wire for which the charge density in the amplification region is higher than for tracks near the wire. Measurements with gamma-sources of different energy $\left({ }^{55} \mathrm{Fe}: 5.9 \mathrm{keV},{ }^{241} \mathrm{Am} / \mathrm{Mo}: 17 \mathrm{keV}\right)$ showed that the streamer probability increases with this charge density [16]. Therefore the streamer fractions measured for distant tracks can be considered as an upper limit for all tracks.

Figure 11a shows the fraction of streamer pulses as a function of the gas gain for different wire diameters. Figure 11b shows the variation of streamer rate with wire diameter. For the three thinner wires there is a tendency to an increase of streamer fraction with increasing wire diameter, which was also reported by other groups about other gases (e.g. [17]); for the $160 \mu \mathrm{m}$ wire however this tendency seems not to hold anymore. We have no explanation why the streamer probability has a maximum at a wire diameter somewhere between $70 \mu \mathrm{m}$ and $160 \mu \mathrm{m}$.

The important conclusion for our purpose is that streamer tendency does not provide 

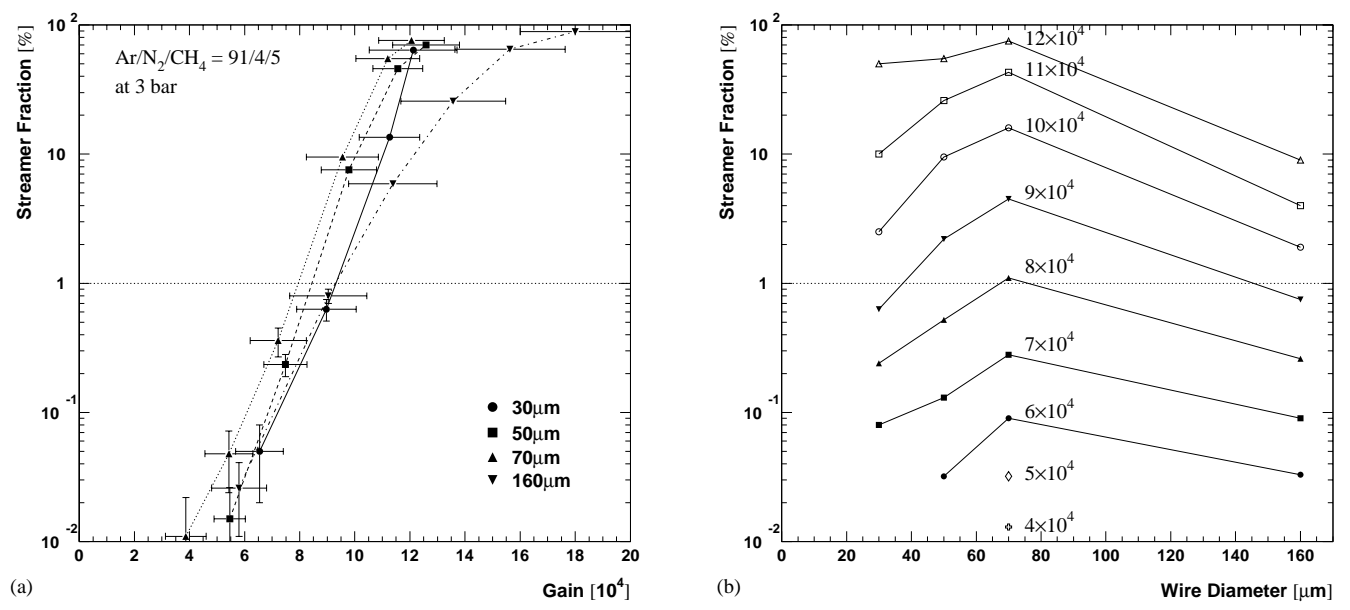

Figure 11: a: Fraction of streamer pulses as a function of the gas gain for different wire diameters. b: Fraction of streamer pulses as a function of the wire diameter for different gas gains. The values have been interpolated from the measurements shown in (a). The connecting lines are drawn to guide the eye.

a strong selection argument for the wire diameter. At gas gains below $4 \times 10^{4}$ - the relevant domain for ATLAS drift tubes - no streamers were observed at all. Given our statistics, at $2 \times 10^{4}$ the upper limit for the streamer rate is $10^{-4}$ with a confidence level of $90 \%$ for all wire diameters.

\section{Conclusion}

Based on the results presented above and considering the requirement of maximum mechanical strength, the ATLAS Muon Collaboration has decided to use wires with $50 \mu \mathrm{m}$ diameter in the MDT chambers. Above that diameter the resolution starts to deteriorate, while for lower diameters there is no significant improvement.

The hit efficiency, $3 \sigma$-efficiency and streamer rate do not provide an argument in favour of any wire diameter:

The hit efficiency is fully determined by the ionization properties of the argonbased gas at a pressure of 3 bar and by effects near the aluminium walls. It has a level of practically $100 \%$.

The $3 \sigma$-efficiency which quantifies the importance of non-gaussian tails, is determined by the delta-ray probability and - near the wire - by the statistical distribution of ionization along the track.

At the baseline gas gain of $2 \times 10^{4}$ the streamer fraction for $\mathrm{Ar} / \mathrm{N}_{2} / \mathrm{CH}_{4}(91 / 4 / 5)$ is less than $10^{-4}$ in all cases studied. 


\section{References}

[1] ATLAS Muon Collaboration: Technical Design Report, CERN/LHCC 97-22, June 1997.

[2] N.P. Hessey: The precision drift chambers for the ATLAS Muon Spectrometer, NIM A 419 (1998) 326-330.

[3] W. Riegler et al.: Resolution Limits of Drift Tubes, NIM A 443/1 (2000) 156-163.

[4] M. Aleksa, W. Riegler: Non-Linear MDT Drift Gases like $\mathrm{Ar} / \mathrm{CO}_{2}$, ATLAS note ATLMUON-98-268, CERN 1998.

[5] M. Deile et al.: Test Beam Studies of Gas Mixtures for Drift Tubes, ATLAS note ATL-MUON-96-122, CERN 1996.

[6] Common base preamplifier IO354-2 and shaping amplifier IO532-01, Instrumentation Division, Brookhaven National Laboratory, Upton, New York, 1973.

[7] P. Rewiersma: The L3 Wire-Amplifier, Type NH19-6112, NIKHEF 1986.

[8] M. Deile, J. Dubbert, N.P. Hessey, A. Staude: ODYSSEUS - a Silicon Telescope for Test Beam Experiments, System Description and User Manual for the Data Analysis, ATL-COM-MUON-99-015, CERN 1999, available on the WWW-page: http://www.etp.physik.uni-muenchen.de/atlas/testbeam.html

[9] J. Dubbert, Bestimmung der Ortsauflösung von Hochdruckdriftrohren für das MyonSpektrometer des ATLAS-Experimentes, diploma thesis, LMU Munich, August 1996

[10] M. Deile, N. Hessey, A. Staude: MDT Track Fitting with the Maximum Likelihood Method, ATLAS Note ATL-MUON-98-240, CERN 1998.

[11] R. Veenhof: GARFIELD, a drift chamber simulation program, Version 6.29, CERN.

[12] I. Smirnov: HEED, program to compute energy loss of fast particles in gases, Version 1.01, CERN

[13] S. Biagi: MAGBOLTZ, a program to compute gas transport parameters, Version 1.16, CERN.

[14] W. Blum, L. Rolandi: Particle Detection with Drift Chambers, Springer Verlag, 1957.

[15] ATLAS Technical Proposal, CERN/LHCC 94-43, LHCC/P2, 1994.

[16] M. Deile: Optimierung der Operationsparameter von Hochdruckdriftrohren für ATLAS, diploma thesis, LMU Munich, March 1996

[17] I.R. Boyko et al.: Investigation of the ratio between streamer and proportional signals in drift tubes, ATLAS note ATL-MUON-95-088, CERN 1995. 